\title{
A medication workshop for patients with schizophrenia living in community care homes
}

Jennifer Perry, Susie Lingwood, Bashir Khaver - Barnet, Enfield and Haringey Mental Health Trust

\begin{abstract}
Psychoeducation for patients with schizophrenia can improve a range of outcomes. Our aim was to test a workshop intervention enabling service users to learn more about mental illness and their medication. A Quality Improvement Project was undertaken to create a workshop for patients with a diagnosis of schizophrenia within the Haringey Community Rehabilitation Team (HCRT). The response was tested using anonymous questionnaires after each workshop. We held ten workshops (total of 47 participants), after which $83 \%$ of patients felt that the workshop had helped them to understand more about their mental health, $77 \%$ felt they were able to understand the purpose of their medication, $79 \%$ felt they were able to understand the side effects, and $70 \%$ felt they could have a say in prescribing. Objectives for the Quality Improvement Project were met. This workshop will be used for other patients within the HCRT and is transferable to other community mental health teams.
\end{abstract}

\section{Problem}

The Haringey Community Rehabilitation Team (HCRT) is within Barnet, Enfield and Haringey Mental Health Trust (BEH-MHT). This team looks after service users who have a psychotic illness, the vast majority of whom have a diagnosis of schizophrenia. These service users live in community residential care homes as they require additional support to live and function in a community setting. There are over 60 residential care homes for people with mental health problems in Haringey; the team looks after approximately 250 patients who are based in these establishments.

\section{Background}

Schizophrenia can be a severe and chronic illness which is often associated with poor compliance with treatment (1). Non adherence is significantly associated with an increased risk of relapse, hospitalisation and suicide attempts (2). It can be the result of a variety of different factors, one of the most important being a lack of insight (3). Other factors may include the stigma of taking medications, adverse drug reactions, forgetfulness, and a lack of social support (4). One method of enhancing compliance with antipsychotics is psychoeducation (5).

The National Institute for Health and Clinical Excellence (NICE) defines psychoeducation as 'the provision of information and advice about a disorder and its treatment. It usually involves an explanatory model of the symptoms and advice on how to cope with or overcome the difficulties a person may experience. It is usually of brief duration, instigated by a healthcare professional, and supported by the use of written materials' (6). A Cochrane review undertaken in February 2010 showed that psychoeducation appeared to reduce relapse, readmission, and encourage medication compliance, as well as reduce the length of hospital stay (1). Brief group psychoeducation has been shown to improve quality of life (7).

\section{Baseline}

BEH-MHT measures patient satisfaction through a Patient Experience Questionnaire. The questionnaire asks three questions:

1)Do staff explain the purpose of medication to you in an understandable way?

2)Do staff explain the possible side effects of medication to you in an understandable way?

3)Do you have a say in your medication choice?

The questionnaire is distributed by care coordinators and doctors to all service users, with a target that each individual should complete it at least once per year. During a six month period (November 2011-April 2012) 65 questionnaires were completed by the patients of the HCRT. A review of the responses highlighted a number of issues. $45 \%$ of patients felt that the side effects of their medication were not always explained to them in an understandable way and $60 \%$ of patients felt that they never, or only sometimes, had a say in their medication choice. This highlighted that there was inconsistency in the provision of information about medication. Only $34 \%$ of service users felt that they always had a say in their medication choice.

See supplementary file: Medication Workshop Results Table.doc

\section{Design}

A PowerPoint presentation was prepared for the workshop which covered the topics of mental illness, schizophrenia and medication. The workshop was designed to be very interactive where attendees would be invited to answer and ask questions and to share their experiences. The session was designed to last $1 \frac{1}{2}$ hours in total 
with a ten minute break in the middle.

At the beginning there was a seven minute warm-up where the facilitators introduced themselves and explained the purpose of the group. Group rules and boundaries were then established (confidentiality, respect for each other's opinions, turn taking etc). The service users were then asked to introduce themselves in turn and say what they would like to get out of the workshop.

The first half of the workshop lasted 30 minutes and focused on mental illness, looking at its definition and exploring how common it is. The workshop then focused on schizophrenia; in particular the definition, epidemiology, symptoms, causes and treatment of the illness. This first half of the workshop gave some background and an introduction to the topic of medication.

Following a ten minute break, the second half of the workshop lasted 40 minutes. It started off with a coloured card exercise where service users were asked to match up medications to the mental illness in which they are most commonly used. The idea behind this was to illustrate that there are a variety of different medications which can be used to treat different mental illnesses. The workshop went on to look at the different types of medication which could be used in schizophrenia, focusing in particular on antipsychotics. The workshop then examined how these drugs work, the different types of antipsychotics that are used, their side effects, their benefits and the potential risks involved if patients don't take their medication. The final part of the workshop focused on how service users could have a say when it came to making decisions about their medication treatment. This highlighted the importance of informing care co-ordinators, key workers or doctors if they had any issues with their medication. The workshop looked at the pros and cons of taking medication and illustrated the importance of finding the best medication for each service user. The presentation looked at some of the options available if service users were not happy with their medication following a discussion with their doctor. For example, a doctor might consider adding in a medication such as procyclidine if their patient were experiencing extra-pyramidal side effects.

At the end there was a three minute closing where service users were thanked for their contributions. During this time information leaflets and anonymous feedback questionnaires were distributed. Service users were also given the details of additional resources they could use if they wanted to find out further information on mental illness or medication.

The anonymous feedback questionnaires asked a variety of questions to see if the workshop objectives had been met. Service users were invited to give their comments and suggestions for improvements which could be made to the workshop. As a result the structure and content of the workshop was modified and improved after each session using the feedback from the questionnaires.

A junior doctor (JP) was identified as the main facilitator for the workshops. She met with the HCRT manager in May 2012 to identify dates and care homes for the medication workshops to take place in. Different team members were identified to co-facilitate each workshop. 12 workshops were identified to take place from May-July 2012. Each care home manager provided a list of their residents with a diagnosis of schizophrenia who would be suitable to invite. We wanted to include as many people as possible in the workshops, however those service users whose mental states were considered too unstable by the care home/HCRT and would risk disrupting the group were excluded. Invitations were sent out to these service users explaining the details and purpose of the workshop. It was made explicitly clear to care home managers that their residents should come to the workshop of their own volition and should have a genuine interest in finding out more about schizophrenia and the medication used to treat it. In terms of group size we advised that this should be between two to eight people with priority given to service users under the HCRT (at some of the residential care homes there were service users who were looked after by different community mental health teams).

The materials required for the workshops were a laptop, projector, coloured cards, feedback questionnaires, pens and patient information leaflets.

A protocol was written explaining how the workshops were set up and run. A copy of the powerpoint presentation was made available to the team on the Trust's shared computer drive. Junior doctors on the HCRT rotate every six months and it was felt that each new doctor should continue to take leadership in the workshops with the support of the team and Consultant. The medication workshop was taken to the local clinical governance meetings to help ensure longevity of the project.

\section{Strategy}

The 'plan' phase was started by stating that the goal of the medication workshop was to expand patient's knowledge and understanding of the medication used in the treatment of schizophrenia. Our hypothesis was that at least $70 \%$ of service users would meet our objectives and that we would test the intervention using anonymous questionnaires. In the 'do' phase the workshop was carried out and feedback questionnaires were distributed to assess the intervention. In the 'study' phase the feedback from the workshop questionnaires was analysed. The 'act' phase consisted of determining what modifications should be made to subsequent workshops as a result of the service user questionnaire feedback and from the facilitator's experience of the workshops. A total of ten PDSA cycles were carried out (one after each workshop) and a number of changes were implemented to the workshops which included; giving a definition of what mental illness is, giving more information on the sexual side effects of drugs and giving more information on drugs used in schizophrenia which are not antipsychotics (e.g. antidepressants).

See supplementary file: PDSA Cycle medication workshop.doc

\section{Post-Measurement}

From 10th May - 31st July 2012, ten workshops were carried out for a total of 47 patients, 39 of whom were under the care of the HCRT. 
These workshops covered twenty of the residential care homes in Haringey. The results from the feedback questionnaires showed that the patients who attended the workshop gave it an average score of $8.3 / 10$ for usefulness (0-Not useful, 10-very useful).

$83 \%(39 / 47)$ of patients felt that the workshop had helped them to understand more about their mental health. $77 \%(36 / 47)$ of patients felt that from this workshop they were able to understand the purpose of their medication. $79 \%$ (37/47) of patients felt that they were able to understand what the side effects of their medications were from this workshop. $70 \%$ (33/47) patients felt they could have a say in their medication from this workshop. Comments on the workshops made by service users were generally positive; people reported to find them 'useful', 'helpful' and 'interesting'.

See supplementary file: Medication Workshop Results Table.doc

\section{Lessons and Limitations}

A difficulty that we encountered was that two of the planned workshops had to be cancelled due to miscommunication between the HCRT and the care homes. This meant that only ten workshops were carried out instead of the twelve which were originally identified. The lesson learnt here was the importance of good communication between the care homes and HCRT staff.

Another lesson learnt was that service user feedback is very important. Using the PDSA cycle, which encouraged an ongoing process of improvement, allowed us to develop a medication workshop which was tailored to the patients' needs. There were a number of challenges to our quality improvement project. $17 \%$ $(8 / 47)$ of the patients who attended these workshops were not under the care of the HCRT which meant that it was more difficult to compare our workshop results to the original Patient Experience Questionnaire results (November 2011-April 2012). Another limitation was that there was no way of identifying whether these workshops had led to an improvement in the results of the medication questions from the Patient Experience Survey. This is because the Patient Experience Questionnaires are given to service users at different care homes on a sporadic basis and it is not possible to identify which care homes the patients are from because the forms are anonymous. Another limitation was that the feedback questionnaires only tested people's attitudes towards medication and it did not test their knowledge or look to see if their behaviour changed as a result of the workshop.

\section{Conclusion}

This medication workshop will continue to be used for patients in the remaining care homes under the HCRT. This workshop is transferable; it could be used in other Community Mental Health Teams (CMHTs) and it could be run by a variety of different healthcare professionals including nurses, pharmacists and doctors.

The HCRT is in the process of setting up further psychoeducation workshops for service users, including a Dual Diagnosis Substance Misuse Workshop. Suggestions for further groups have included a healthy eating/lifestyle group and a medication workshop for the staff of the care homes.

The results of the feedback questionnaires show that our objectives were all met at $70 \%$ or higher with regards to patients understanding more about their mental health, understanding the purpose and the side effects of their medication and understanding that they can have a say when it comes to their medication. The results from the workshop show an improvement in the medication objectives compared with the original results from the Patient Experience Questionnaires (November 2011-April 2012). The feedback was overall positive with people generally finding the workshop useful. A number of the suggestions advised by patients and a number of improvements which were a result of the facilitators' experience of the workshops were incorporated into the project. This was done using the PDSA cycle methodology allowing for an ongoing process of improvement.

Psychoeducation is associated with a variety of benefits for patients which include reducing relapse and readmission rates, encouraging medication compliance, reducing the length of hospital stay and improving patient's quality of life $(1,7)$. This workshop yielded positive results, required few resources and was low in cost to implement which should make it attractive to both clinicians and managers (8).

\section{References}

1) Xia J, Merinder LB, Belgamwar MR, Psychoeducation for Schizophrenia (2011). Cochrane Database Systematic Review, Jun 15,6

2) Novick D, Haro JM, Suarez D, Perez V, Dittmann RW, Haddad PM, (2010). Predictors and clinical consequences of non-adherence with antipsychotic medication in the outpatient treatment of schizophrenia, Psychiatry Research, Apr 30; 176(2-3), 109-13 3) Dassa D, Boyer L, Benoit M, Bourcet S, Raymondet P, Bottai T, (2010). Factors associated with medication non-adherence in patients suffering from schizophrenia: a cross-sectional study in a universal coverage health-care system, The Australian and New Zealand journal of psychiatry, October 44(10), 921-8

4) Hudson TJ, Owen RR, Thrush CR, Han X, Pyne JM, Thapa P, Sullivan $G$, (2004). A pilot study of barriers to medication adherence in schizophrenia, Journal of Clinical Psychiatry, Feb vol./is. 65/2, 211-6

5) Velligan DI, Weiden PJ, Sajatovic M, Scott J, Carpenter D, Ross R, Docherty JP, (2010). Strategies for addressing adherence problems in patients with serious and persistent mental illness: recommendations from the expert consensus guidelines, Journal of Psychiatric Practice, Sep, 16(5), 306-24

6) CG123 Nice Guidance: Common mental health disorders: Identification and pathways to care, glossary of terms,( 2011). May, http://guidance.nice.org.uk/CG123/NICEGuidance/pdf/English (accessed 2/7/12) page 54.

7) Bechdolf A,Knost B,Nelson B,Schneider N,Veith V,Yung AR,Pukrop, (2010). Randomized comparison of group cognitive behaviour therapy and group psychoeducation in acute patients with schizophrenia: effects on subjective quality of life, The Australian and New Zealand journal of psychiatry, February vol 


\section{BMJ Quality Improvement Reports}

$44 / 2,144-50$

8) Pekkala ET, Merinder LB, Psychoeducation for schizophrenia

(Review), (2002). Cochrane Database Systematic Review, 2

\section{Acknowledgements}

Staff working in the Haringey Community Rehabilitation Team: Shireen Hussain-Roy (Team manager), Edward Lander, Agnes Tekyi, Patrick Mamattah, Grace Ogundayo, Nick Mangwana, Adam Madarbux, Maxine Richardson, Greg Murray, Maura Duffy

Other Staff from Barnet, Enfield and Haringey Mental Health Trust: Dr Nicole Eady (Psychiatry Core Trainee), Dr Guy Thompson

(Psychiatry Specialist Registrar), Dr Sujeet Jaydeokar (Consultant Psychiatrist), Professor Ikkos (Director of Medical Education), Dr Liz Sampson (Consultant Psychiatrist, UCL Senior Clinical Lecturer) 